\title{
The challenges of mouth cancer
}

\section{awareness in a post-COVID world} increasingly resistant to change

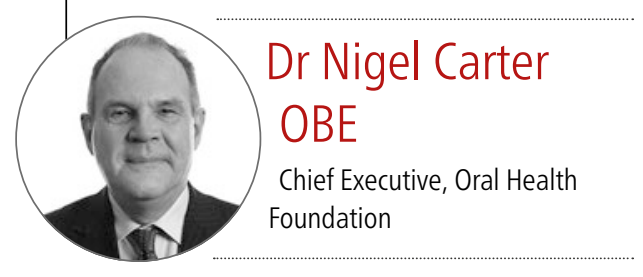

his November our charity marks 20 years of Mouth Cancer Action Month. For two decades we Chave prioritised mouth cancer awareness in a bid to get more mouth cancers diagnosed earlier and reduce the number of lives lost to the disease. During this time, we have been proud to work alongside tens of thousands of dental professionals and organisations, all of who have an equal passion for providing local communities, patients and mouth cancer survivors, with support about a disease that continues to cause harm to so many lives.

Since the very first campaign back in the year 2000, public health messages around the early warning signs of the disease, along with the risk factors, have been a focal point of the initiative. Along with advances in treatment, public awareness of the symptoms and the lifestyle choices associated with the disease, have had a tremendous impact in transforming how much the public know about mouth cancer. Just as it has done for so many other cancers and diseases.

But there might be an unexpected roadblock ahead.

The pandemic has presented dentistry with a whole host of unprecedented clinical and financial challenges. I am in great admiration of the profession, which has excelled in adapting to the changes required to practise during COVID-19, in hugely difficult times.
Despite patients being compliant with what has been asked of them, it feels like we are at a turning point regarding the wider general public. There is a growing resistance to increasingly frequent, and sometimes puzzling, policy directions and restrictions on personal freedoms. Advice, particularly around health and wellbeing, has at times been particularly confusing. Government decisions to implement advice from bodies such as SAGE, have been a rather pick-and-mix selection, leaving many to distrust what is scientifically justified and what can be called into question.

While this concern may seem very specific to COVID-19, there is a real threat that resistance to accept health messages from authoritative bodies could leak over into other areas of health. For the progression of mouth cancer awareness, this would undoubtably be a disaster.

\section{The state of mouth cancer}

Before we begin to discuss the wider impact of any resistance to COVID-19 messaging, let's just take a moment to look at the state of mouth cancer in the UK. Last year, 8,722 people in the UK were diagnosed with mouth cancer. This translates to almost one person every hour. ${ }^{1}$ The number of new cases has almost doubled over the last 20 years and the disease is one of very few cancers which is, sadly, going in the wrong direction. ${ }^{2}$

Mouth cancer is now the $15^{\text {th }}$ most common cancer in the UK and continues to travel up the list of most prevalent cancers. The demographics of the disease have barely changed with time. While more younger people and women are being diagnosed with the disease, mouth cancer is still strongly associated with men and those over the age of $55 .^{2}$

The sites for mouth cancer also remain similar, with mouth cancers more likely to be diagnosed in the tongue - these make up around one in three cases.

Sadly, more than 2,700 people in the United Kingdom lost their life to mouth cancer last year. That's seven people every day. ${ }^{2}$ While awareness of the signs and symptoms, along with risk factors, and more access to dentistry should be leading to earlier diagnosis, far too many cases are caught in the later stages - stage III and IV. Not only does this reduce a person's chances of beating the disease, it also decreases their quality of life after treatment.

\section{Response to COVID-19 health messages}

The UK's confidence in the government's handling of coronavirus is at an all-time low. Only $31 \%$ of Brits now believe the response to COVID-19 has been dealt with well, tumbling by $24 \%$ over the last the last six months. ${ }^{3}$ As you would expect from such data, trust in our leaders has also fallen. Only $31 \%$ of the public now trust Boris Johnson and Matt Hancock's trust has plummeted to just $23 \% .{ }^{4}$ In addition, support for the civil service is down to $29 \%$ while newspaper trust is at $12 \%{ }^{4}$

It is difficult to accurately provide an explanation for such a fall in faith. A population no longer willing to sacrifice their daily lives, the toll of long-standing lockdown, or a loss of belief in the threat of COVID-19, could all be possible scenarios. However, if that is truly the case, then we must question why other European countries with similarly-tough restrictions, like Germany and Italy, have government support ratings sitting at $63 \%$ and $64 \%$, respectively more than double that of our own. ${ }^{3}$

This would suggest that the restrictions themselves are not the issue here, but instead, the manner in which they are being delivered. 
Mouth Cancer Action Month November 2020

Oral Health Foundation

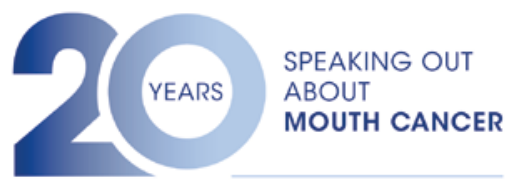

Despite face coverings being mandatory in any number of indoor settings, UK compliance as of October, was $76 \%{ }^{3}$ This means nearly one-in-four are non-compliant. A remarkable number considering the threat we are being told about. Looking at some of the more recent restrictions, $38 \%$ of people do not believe that $10 \mathrm{pm}$ pub curfews and table service, limitations to wedding and funerals, and working from home, makes any difference whatsoever to the spread of the virus. ${ }^{5}$

A lack of trust in not only our leaders, but our mainstream communication outlets, is deeply concerning. It all leaves me with grave concerns about the knock-on effect it could have on mouth cancer.

\section{A tragedy for public health}

The most obvious impact from any fallout from the pandemic is in relation to a resistance of lifestyle choices, namely, smoking, alcohol and HPV - all of which are risk factors for mouth cancer.

In the face of further restrictions to personal movement and limitations placed upon businesses, we have found evidence of a growing backlash to proposals to introduce new health policies. Over the last two years, we have polled UK adults for their support of several new policies on smoking and alcohol. Examples of policy suggestions included plain packaging for alcohol, health warning labels on alcohol, an outright smoking ban, and banning smoking outside where food is served. Out of the 19 policies, 17 of them received less support in October 2020, compared with before the pandemic. Overall, support of these polices fell by around $10 \%$ year-on-year.

Whether this is down to political fatigue from government's seesaw approach to COVID-19, or a reaction to a perceived 'nanny state' is only speculation at this stage. Regardless, public support for new policy is essential for its introduction and without their support, implementing new health policies could become increasingly difficult in the future.

The other element of change we have observed during COVID-19 has been regarding general support for vaccinations.
The HPV vaccine has played an instrumental role in eliminating cervical cancer in young women, while also protecting them from mouth cancer. We were delighted when the vaccination was extended to schoolboys in September 2019 and believe it is the most influential public health measure taken since the smoking ban was introduced in 2007. Latest statistics show that the take-up rate for the HPV vaccine is $84 \%$ - an acceptable number for effectiveness. ${ }^{6}$

However, our research indicates cause for concern. Support for the coronavirus vaccine is as low as $73 \%$ and this has had a direct impact on the number of parents who view the HPV vaccine in a positive manner. A HPV vaccine rate of $73 \%$ would be a tragedy for public health and we must do all we can to make sure acceptance for the vaccine remains high. ${ }^{7}$

It remains that the best way anybody can protect themselves from the disease is to be aware of the risk factors and adjust their lifestyle accordingly. One of the leading causes of mouth cancer is drinking alcohol to excess, linked to around a third of all cases.

Since lockdown, our research has found that $24 \%$ of British adults have increased their drinking habits. Of an even greater concern, $41 \%$ of people who drink more than the government's recommended amount of 14 units a week, have also increased their consumption. ${ }^{7}$ Despite being in a time where many are taking their health more seriously, pockets of the population continue to pursue lifestyle choices that increase their risk of not only mouth cancer, but other alcohol-related diseases.

\section{A positive influence on the public and final thoughts}

Over the last six months, there have been plenty of examples of public disobedience regarding the government's guidance around coronavirus. The motivations behind such resistance varies but is it clear that many have lost faith and trust in the scientific evidence which exists to protect the population's health. It is essential that public health messages around mouth cancer are not so easily dismissed.
Being able to have the general public's support is a privilege that should not be taken for granted, and as the dental profession, we must continue to be an authoritative and reassuring voice in matters of health. This can be achieved with consistent advice that is easy to understand and straight forward to follow. All being on the same page is vital.

During November's Mouth Cancer Action Month, we will be working to advise, educate and engage all people about mouth cancer. We want everybody to be mouthaware and able to identify the early warning signs of mouth cancer. Importantly, we also want to communicate the risk factors of mouth cancer. Individuals must understand their own level of risk, and if cutting down or quitting is not an option, then these groups simply must be more aware to changes in their mouth and be on board with more regular dental visits.

We invite you to be part of this journey we need your help. By visiting the campaign website at www.mouthcancer.org you will be able to download our toolkit and digital resources so that we can help educate patients and local communities online. By working together, we can rebuild the public's trust in health messages and reduce the devastating harm caused by mouth cancer.

\section{References}

1. Oral Health Foundation (2020). State of Mouth Cancer UK Report 2020/21. Available online at: https://www.dentalhealth.org/ thestateofmouthcancer (Accessed October 2020).

2. Oral Health Foundation (2020). 'Mouth Cancer Statistics', online at https://www. dentalhealth.org/Pages/FAQs/Default. aspx? CategoryTitle=mouth-cancer-statistics\&Sub siteTitle=mouthcancer (Accessed October 2020).

3. YouGov (2020). Latest round-up of YouGov's coronavirus survey results. Available online at: https://yougov.co.uk/topics/health/articlesreports/2020/09/02/international-covid-19-trackerupdate-2-september (Accessed October 2020).

4. YouGov (2020). YouGov/Sky Survey Results. Available online at: https://docs.cdn.yougov. com/k6h1g211k5/Sky_CoronaBrexit_201005. pdf (Accessed October 2020).

5. YouGov (2020). YouGov Survey Results. Available online at: https://docs.cdn yougov.com/xw2puh6p0r/Internal_ COVIDRestriction_200922_W.pdf (Accessed October 2020).

6. Public Health England (2019). Human papillomavirus (HPV) vaccination coverage in adolescent females in England: 2018/19'. Available online at: https://assets.publishing. service.gov.uk/government/uploads/system/ uploads/attachment_data/file/851797/HPV_ annual_coverage_report_2018_to_2019.pdf (Accessed October 2020).

7. Oral Health Foundation (2020). Mouth Cancer Action 2020 Survey. 13.10.2020, sample 2,008. Data on file.

https://doi.org/10.1038/s41404-020-0556-8 